\title{
Discovery of anti-2019-nCoV agents from 38 Chinese patent drugs toward respiratory diseases via docking screening
}

Yong-Ming Yan ${ }^{1, \dagger}$, Xin Shen ${ }^{2, \dagger}$, Yong-Kai Cao ${ }^{1}$, Jiao-Jiao Zhang ${ }^{1}$, Yan Wang, ${ }^{2, *}$, Yong-Xian Cheng ${ }^{1, *}$

${ }^{1}$ School of Pharmaceutical Sciences, Shenzhen University Health Science Center, Shenzhen 518060, P.R. China

${ }^{2}$ Center for Translation Medicine Research and Development, Shenzhen Institute of Advanced Technology, Chinese Academy of Sciences, Shenzhen 518055, P.R. China

* To whom correspondence should be addressed: Tel: 86755-2690 2073; E-mail: yxcheng@szu.edu.cn (Y.-X. C.); 86755-2641 7985; E-mail: yan.wang@siat.ac.cn (Y. W.)

${ }^{\dagger}$ These authors contributed equally to this paper.

Running Title: Natural agents against 2019-nCoV by docking screening Keywords: 2019-nCoV, novel coronavirus pneumonia, docking, ACE2, viral main protease 


\section{Abstract}

The 2019 novel coronavirus (2019-nCoV) causes novel coronavirus pneumonia (NCP). Given that approved drug repurposing becomes a common strategy to quickly find antiviral treatments, a collection of FDA-approved drugs can be powerful resources for new anti-NCP indication discoveries. In addition to synthetic compounds, Chinese Patent Drugs (CPD), also play a key role in the treatment of virus related infections diseases in China. Here we compiled major components from 38 CPDs that are commonly used in the respiratory diseases and docked them against two drug targets, ACE2 receptor and viral main protease. According to our docking screening, 10 antiviral components, including hesperidin, saikosaponin A, rutin, corosolic acid, verbascoside, baicalin, glycyrrhizin, mulberroside A, cynaroside, and bilirubin, can directly bind to both host cell target ACE2 receptor and viral target main protease. In combination of the docking results, the natural abundance of the substances, and botanical knowledge, we proposed that artemisinin, rutin, glycyrrhizin, cholic acid, hyodeoxycholic acid, puerarin, oleanic acid, andrographolide, matrine, codeine, morphine, chlorogenic acid, and baicalin (or Yinhuang Injection containing chlorogenic acid and baicalin) might be of value for clinical trials during a 2019-nCov outbreak. 


\section{Introduction}

The 2019 novel coronavirus (2019-nCoV), named as the Wuhan coronavirus [the pneumonia caused by it is now named as novel coronavirus pneumonia (NCP)], is a positive-sense, single-strand RNA coronavirus (1). Up to date, global infections of 2019-nCoV surge past 40,000 (WHO website). Given that drug repurposing is the common strategy to search antiviral treatments, several approved drugs were reported to benefit patients (2). Besides synthetic compounds, natural products, especially Chinese Patent Drug (CPD), also play a key role in the treatment of virus related infections diseases in China. Although the mechanisms of CPDs might be associated with immune regulation, we focus on their antiviral properties. In this study. we compiled major components from 38 CPDs that are commonly used in the respiratory diseases and docked them against two drug targets, ACE2 receptor and viral main protease.

Like severe acute respiratory syndrome-related coronavirus (SARS-CoV), the 2019-nCoV attach to host cells through S protein and angiotensin converting enzyme 2 (ACE2) receptor interaction (3). The catalytic inhibitor of ACE2 receptor is likely to induce a conformational change of ACE2, therefore blocking the interaction between $\mathrm{S}$ protein and ACE2 receptor (4). S protein of 2019-nCoV is not currently available but the structure of ACE2 receptor is well-known (5). Thus ACE2 receptor was selected to quickly identify entry inhibitors of 2019-nCoV using marketed CPDs-derived natural products. 
In addition to entry inhibitors, the replication inhibitors are also good strategies for antiviral drug discovery and development (6). Given that 2019-nCoV is a (+)SS RNA virus, its main protease is likely to be required to mediate viral replication and transcription through extensive cleavage of two replicase polyproteins. Therefore inhibition of viral main protease might block virus replication (7). Up to date, Rao et al reported the crystal structure of $\mathrm{M}$ protease of 2019-nCoV (PDB: 6LU7) and several drug repurposing docking screening studies were reported. We herein docked natural product database to main protease to look for antiviral replication agents.

Due to the limited time and lack of the available 2019-nCoV in hand, it is impossible to develop novel compounds against 2019-nCoV by biological screening. We here used docking screening to identify natural products from marketed CPDs that inhibit both virus entry and replication, therefore providing a potential prevention/treatment alternative against 2019-nCoV.

\section{Material and Methods}

The major components of each herb in the selected 38 CPDs were collected as the ligands, and all the ligands were in PDBQT format. The protein model 1R4L was selected as ACE2 receptor docking model while 6LU7 was selected as M protease docking model. Both PDB files of protein models were fetched from Protein Data Bank. The docking screenings were conducted by using AutoDock Vina v.1.0.2. The docking parameters for AutoDock Vina were kept at their default values. The grid box was $25 \AA$ by $25 \AA$ by $25 \AA$, encompassing the catalytic pocket. The binding modes 
were clustered through the root mean square deviation (RMSD) among the Cartesian coordinates of the ligand atoms.

\section{Results and Discussion}

A total of 38 marketed CPDs (bold line in Table 1) containing 93 herbs used for the treatment of respiratory diseases were selected. Totally we docked 95 components (Table 2) and the top 10 hits were summarized in Table 3. All of them provide good binding affinities against both two targets. The key residues for each ligand binding were also summarized in Table 4.

Analysis of the results from Table 3, it was found that the top 10 antiviral components are hesperidin, saikosaponin A, rutin, corosolic acid, verbascoside, baicalin, glycyrrhizin, mulberroside A, cynaroside, and bilirubin, and their binding sites toward 6LU7 and 1R4L are listed in Table 4. A close analysis found that 19 compounds directly bind to ACE2 receptor with high affinities (docking score <-10 kcal/mol), these compounds are hesperidin, saikosaponin A, mulberroside A, rutin, bilirubin, verbascoside, vincetoxicoside $\mathrm{B}$, baicalin, prim- $O$-glucosylcimifugin, corosolic acid, cynaroside, orientin, corynoline, astragaloside A, protostemonine, ilexgenin A, amygdalin, paeoniflorin, and ursolic acid (Table 2). Whereas, in M protease docking screening, 12 phytochemicals, rutin, glycyrrhizin, dipsacoside B, saikosaponin A, corosolic acid, puerarin, morusin, hesperidin, polyphyllin I, verbascoside, baicalin, and cynaroside have been identified as potential M protease inhibitors (docking score $\leq-8.4 \mathrm{kcal} / \mathrm{mol}$ ), indicating their potential for 2019-nCov. Notably, artemisinin, 
berberine, rutin, glycyrrhizin, chlorogenic acid, baicalin, cholic acid, hyodeoxycholic acid, puerarin, oleanic acid, andrographolide, catalpol, matrine, codeine, morphine, caffeic acid, $\alpha$-asarone, $\alpha$-pinene, and taurine are commercially available with good supply (already marketed drugs). However, a combination of their docking results, natural abundance, and traditional knowledge from their source herbs allows us to recommend artemisinin, rutin, glycyrrhizin, chlorogenic acid, baicalin, cholic acid, hyodeoxycholic acid, puerarin, oleanic acid, andrographolide, matrine, codeine, and morphine for clinical trials during a 2019-nCov outbreak. Yinhuang Injection, a marketed drug in China, might be also worth recommendation because it is mainly composed of chlorogenic acid and baicalin. In addition, the results of Table 5 in combination of the literature data indicate the natural sources of these active compounds with relatively high content. Basically, around 34 compounds are present in natural sources more than $1 \%(\mathrm{~g} / \mathrm{g})$, which are respectively hesperidin, baicalin, glycyrrhizin, puerarin, amygdalin, paeoniflorin, berberine, arctiin, forsythiaside A, chlorogenic acid, geniposide, tectoridin, timosaponin BII, dryocrassin, oleanic acid, genistein, trisalbaspidin ABA, daidzein, andrographolide, rosmarinic acid, quercetin (source plant: Sophorae Flos), curcumin (source plant: Curcumae Longae Rhizoma), dipsacoside B (source plant: Lonicerae Dasystylae Flos), rutin (source plant: Potentilla chinensis), and harpagide (source plant: Ajuga pantantha). This natural abundance information in combination with the docking results and the medicinal values of the source herbs suggests that the plants or herbs or their extracts with the above enriched active compounds might be valuable for fighting against 
2019-nCoV. Although the content of magnolol, lobetyolin, pulegone, citrulline, L-menthol, 6-gingerol, catalpol, caffeic acid, and trans-cinnamaldehyde is also more than $1 \%$, it might be not potential either from their docking resluts or botanical knowledge (Table 5). Despite that the other herbs or CPDs are not found to be active toward 2019-nCoV, this doesn't mean that they are not useful for NCP because only limited compounds in herbs were selected which couldn't exclude more compounds or their analogues in herbs of CPDs are active. In addition, the principles of formulating Chinese herbal prescription include eliminating evil and strengthening the body resistance, therefore, we couldn't exclude that these CPDs do work against NCP via regulating immune system.

\section{Acknowledgments}

This study was supported by the National Science Fund for Distinguished Young Scholars (81525026) and National Natural Science Foundation of China (81903875).

\section{Competing interest statement}

The authors declare no conflict of interest.

\section{References}

1. Huang CL, Wang YM, Li XW, Ren LL, Zhao JP, Hu Y, Zhang L, Fan GH, Xu, JY, Gu, XY, Cheng ZS, Yu, T, Xia JA, Wei A, Wu, WJ, Xie XL, Yin W, Li H, Liu, M, Xiao Y, Gao H,Guo L, Xie JG, Wang GF, Jiang RM, Gao ZC, Jin Q, Wang JW, Cao B. Clinical features of patients infected with 2019 novel coronavirus in Wuhan, China. Lancet, 2020; doi: 10.1016/S0140-6736(20)30183-5. 
2. Wang ML, Cao RY, Zhang LK, Yang XL, Liu J. Xu MY, Shi ZL, Hu ZH, Zhong W, Xiao GF. Remdesivir and chloroquine effectively inhibit the recently emerged novel coronavirus (2019-nCoV) in vitro. Cell Res., 2020; doi: 10.1038/s41422-020-0282-0.

3. Wan YS, Shang J, Graham R, Baric RS, Li F. Receptor recognition by novel coronavirus from Wuhan: An analysis based on decade-long structural studies of SARS. J. Virol., 2020; doi: 10.1128/JVI.00127-20.

4. Du LY, He YX, Zhou YS, Liu SW, Zheng BJ, Jiang SB. The spike protein of SARS-CoV -a target for vaccine and therapeutic development. Nat. Rev. Microbiol., 2009; 7(3): 226-236.

5. Towler P, Staker B, Prasad SG, Menon S, Tang J, Parsons T, Ryan D, Fisher M, Williams D, Dales NA, Patane MA, Pantoliano MW. ACE2 X-ray structures reveal a large hinge-bending motion important for inhibitor binding and catalysis. J. Biol. Chem., 2004; 279(17):17996-8007.

6. Clercq ED. Strategies in the design of antiviral drugs. Nat. Rev. Drug Discov., 2002; 1: $13-25$.

7. Wit ED, van Doremalen NV, Falzarano D, Munster VJ. SARS and MERS: recent insights into emerging coronaviruses. Nat. Rev. Microbiol., 2016; 14: 523-534. 
Table 1. Commercial names of 38 Chinese patent drugs (CPDs)

\begin{tabular}{|c|c|c|c|}
\hline No. & CPDs & No. & CPDs \\
\hline 1 & Fengre Ganmao Granules & 20 & Kangbingdu Capsules \\
\hline 2 & Xiaochaihu Granules & 21 & Fufang Banlangen Granules \\
\hline 3 & Qingkailing Capsules & 22 & $\begin{array}{l}\text { Ganmao Shufeng } \\
\text { Capsules/Granules }\end{array}$ \\
\hline 4 & Jinlianhua Capsules & 23 & Ganmao Qingre Granules \\
\hline 5 & Zhongganling Capsules & 24 & Fufang Jinyinhua Granules \\
\hline 6 & $\begin{array}{l}\text { Lianhua Qingwen } \\
\text { Capsules/Granules }\end{array}$ & 25 & Yinqiao Jiedu Pills/Granules \\
\hline 7 & Lanqin Oral Solution & 26 & Vitamin C Yinqiao Tablets \\
\hline 8 & Qingwen Jiedu Tablets & 27 & $\begin{array}{l}\text { Fufang Yinqiao Anfen } \\
\text { Capsules }\end{array}$ \\
\hline 9 & Fangfeng Tongsheng Pills & 28 & Xiasangju Granules \\
\hline 10 & $\begin{array}{l}\text { Shuanghuanglian Oral } \\
\text { Solution }\end{array}$ & 29 & $\begin{array}{l}\text { Vitamin C Effervescent } \\
\text { Tablets }\end{array}$ \\
\hline 11 & $\begin{array}{l}\text { Huoxiang Zhengqi Oral } \\
\text { Solution }\end{array}$ & 30 & Xiaoer Ganmao Granules \\
\hline 12 & Huoxiang Zhengqi Capsules & 31 & Banlangen Granules \\
\hline 13 & Maxing Zhike Syrup & 32 & Qingkailing Oral Solution \\
\hline 14 & Choulingdan Oral Solution & 33 & Yinqiao Jiedu Granules \\
\hline 15 & Erding Capsules & 34 & $\begin{array}{l}\text { Fufang Yinqiao Anfen } \\
\text { Vitamin C Tablets }\end{array}$ \\
\hline 16 & Zhiganjia Granules & 35 & Ganmao Soft Capsules \\
\hline 17 & Kanggan Granules & 36 & Fenghan Ganmao Granules \\
\hline 18 & Kangbingdu Granules & 37 & Qiangli Pipa Syrup \\
\hline 19 & Kangbingdu Oral Emulsion & 38 & Fufang Anwanan Tablets \\
\hline
\end{tabular}


Table 2. Appendix total docking ranking

\begin{tabular}{|c|c|c|c|}
\hline \multirow{2}{*}{ Ligand } & \multicolumn{3}{|c|}{ Docking score (kcal/mol) } \\
\hline & 6LU7 & 1R4L & SUM \\
\hline Hesperidin & -8.5 & -11.4 & -19.9 \\
\hline Saikosaponin A & -8.8 & -11 & -19.8 \\
\hline Rutin & -8.9 & -10.7 & -19.6 \\
\hline Corosolic acid & -8.8 & -10.2 & -19 \\
\hline Verbascoside & -8.4 & -10.6 & -19 \\
\hline Baicalin & -8.4 & -10.5 & -18.9 \\
\hline Glycyrrhizin & -8.9 & -9.9 & -18.8 \\
\hline Mulberroside A & -7.7 & -11 & -18.7 \\
\hline Cynaroside & -8.4 & -10.2 & -18.6 \\
\hline Bilirubin & -7.8 & -10.7 & -18.5 \\
\hline Vincetoxicoside B & -7.9 & -10.6 & -18.5 \\
\hline Morusin & -8.6 & -9.8 & -18.4 \\
\hline Puerarin & -8.6 & -9.8 & -18.4 \\
\hline Orientin & -8.1 & -10.2 & -18.3 \\
\hline Cynancersicoside A & -8.3 & -9.9 & -18.2 \\
\hline Protostemonine & -8.1 & -10.1 & -18.2 \\
\hline Amygdalin & -8.1 & -10 & -18.1 \\
\hline Ilexgenin A & -7.9 & -10.1 & -18 \\
\hline Prim-O-glucosylcimifugin & -7.6 & -10.4 & -18 \\
\hline Corynoline & -7.7 & -10.2 & -17.9 \\
\hline Astragaloside A & -7.6 & -10.2 & -17.8 \\
\hline Paeoniflorin & -7.7 & -10 & -17.7 \\
\hline Polyphyllin I & -8.5 & -9.1 & -17.6 \\
\hline Nodakenin & -7.9 & -9.6 & -17.5 \\
\hline Tectoridin & -7.9 & -9.5 & -17.4 \\
\hline Ursolic acid & -7.4 & -10 & -17.4 \\
\hline
\end{tabular}




\begin{tabular}{|c|c|c|c|}
\hline Swertiajaponin & -8 & -9.4 & -17.4 \\
\hline Berberine & -7.5 & -9.7 & -17.2 \\
\hline Timosaponin BII & -7.7 & -9.4 & -17.1 \\
\hline Dryocrassin & -7.4 & -9.5 & -16.9 \\
\hline Columbianadin & -7.2 & -9.6 & -16.8 \\
\hline Arctiin & -7.3 & -9.5 & -16.8 \\
\hline Oleanic acid & -7.4 & -9.3 & -16.7 \\
\hline Luteolin & -7.6 & -9.1 & -16.7 \\
\hline Quercetin & -7.7 & -9 & -16.7 \\
\hline Forsythiaside A & -7.6 & -9.1 & -16.7 \\
\hline Radix isatidis $\mathrm{A}$ & -7.6 & -9.1 & -16.7 \\
\hline Genistein & -7.5 & -9.1 & -16.6 \\
\hline Indirubin & -7.3 & -9.3 & -16.6 \\
\hline Curcumin & -7 & -9.5 & -16.5 \\
\hline Trisalbaspidin ABA & -7.2 & -9.3 & -16.5 \\
\hline Artemisinin & -7.3 & -9.1 & -16.4 \\
\hline Emodin & -7.2 & -9.2 & -16.4 \\
\hline Cholic acid & -7 & -9.3 & -16.3 \\
\hline Hyodeoxycholic acid & -7 & -9.3 & -16.3 \\
\hline Daidzein & -7.4 & -8.8 & -16.2 \\
\hline Xanthiside & -7.3 & -8.9 & -16.2 \\
\hline Chlorogenic acid & -7.3 & -8.8 & -16.1 \\
\hline Verbenalin & -7.4 & -8.7 & -16.1 \\
\hline Poricoic acid A & -6.9 & -9.2 & -16.1 \\
\hline Andrographolide & -6.9 & -8.8 & -15.7 \\
\hline Dipsacoside B & -8.9 & -6.7 & -15.6 \\
\hline Codeine & -7 & -8.5 & -15.5 \\
\hline Rosmarinic acid & -7 & -8.4 & -15.4 \\
\hline Notopterol & -7 & -8.4 & -15.4 \\
\hline
\end{tabular}




\begin{tabular}{|c|c|c|c|}
\hline Harpagide & -7 & -8.3 & -15.3 \\
\hline Imperatorin & -7.1 & -8.2 & -15.3 \\
\hline Papaverine & -6.9 & -8.3 & -15.2 \\
\hline Geniposide & -6.7 & -8.5 & -15.2 \\
\hline Catalpol & -7.1 & -7.9 & -15 \\
\hline Salidroside & -6.9 & -7.9 & -14.8 \\
\hline Morphine & -6.6 & -8.1 & -14.7 \\
\hline Atractylenolide I & -6.3 & -8.2 & -14.5 \\
\hline Magnolol & -6.4 & -7.9 & -14.3 \\
\hline Lobetyolin & -6.4 & -7.7 & -14.1 \\
\hline Matrine & -6.1 & -7.9 & -14 \\
\hline Pterodontic acid & -6 & -7.7 & -13.7 \\
\hline Isoevodionol & -6.2 & -7.1 & -13.3 \\
\hline Esculetin & -6.2 & -6.9 & -13.1 \\
\hline Platycodin D & -7.5 & -5.5 & -13 \\
\hline Scopoletin & -5.8 & -6.8 & -12.6 \\
\hline Dhelwangin & -5.2 & -7.1 & -12.3 \\
\hline Caffeic acid & -5.7 & -6.5 & -12.2 \\
\hline Ferulic acid & -5.4 & -6.5 & -11.9 \\
\hline 6-Gingerol & -4.8 & -6.6 & -11.4 \\
\hline $\mathrm{L}(+)$-Ascorbic acid & -5.1 & -6.1 & -11.2 \\
\hline Atractylodin & -4.9 & -6.3 & -11.2 \\
\hline Ephedrine & -5.1 & -6.1 & -11.2 \\
\hline Pulegone & -4.9 & -6.2 & -11.1 \\
\hline$\alpha$-Asarone & -5.1 & -5.9 & -11 \\
\hline Coumalic acid & -4.9 & -5.9 & -10.8 \\
\hline Citrulline & -4.9 & -5.8 & -10.7 \\
\hline Linolenic acid & -4.6 & -6.1 & -10.7 \\
\hline Amantadine Hydrochloride & -4.4 & -6.2 & -10.6 \\
\hline
\end{tabular}




\begin{tabular}{lccc} 
L-Menthol & -4.7 & -5.7 & -10.4 \\
trans-Cinnamaldehyde & -4.4 & -5.7 & -10.1 \\
$\beta-$-Pinene & -4.3 & -5.7 & -10 \\
Arecoline & -4.6 & -5.4 & -10 \\
Glutamic acid & -4.5 & -5.3 & -9.8 \\
$\alpha-$ Pinene & -4.1 & -5.6 & -9.7 \\
Tetramethyl pyrazine & -4.5 & -5.1 & -9.6 \\
Succinic acid & -4.4 & -4.9 & -9.3 \\
Decanoy acetaldehyde & -3.9 & -4.9 & -8.8 \\
Taurine & -3.7 & -4.2 & -7.9 \\
Betaine & -3.5 & -4.1 & -7.6 \\
\hline
\end{tabular}

Table 3. Natural products from CPDs docking results

\begin{tabular}{llll}
\hline \multirow{1}{*}{\multicolumn{1}{c}{ Ligand }} & \multicolumn{3}{c}{ Docking score (kcal/mol) } \\
& 6LU7 & 1R4L & SUM \\
\hline Hesperidin & -8.5 & -11.4 & -19.9 \\
Saikosaponin A & -8.8 & -11 & -19.8 \\
Rutin & -8.9 & -10.7 & -19.6 \\
Corosolic acid & -8.8 & -10.2 & -19 \\
Verbascoside & -8.4 & -10.6 & -19 \\
Baicalin & -8.4 & -10.5 & -18.9 \\
Glycyrrhizin & -8.9 & -9.9 & -18.8 \\
Mulberroside A & -7.7 & -11 & -18.7 \\
Cynaroside & -8.4 & -10.2 & -18.6 \\
Bilirubin & -7.8 & -10.7 & -18.5 \\
\hline
\end{tabular}


Table 4. Key residues for potential inhibitors binding

\begin{tabular}{|c|c|c|}
\hline \multirow{2}{*}{ Ligand } & \multicolumn{2}{|c|}{ Key residues } \\
\hline & 6LU7 & 1R4L \\
\hline Hesperidin & $\begin{array}{l}\text { Gly143, Ser144, Cys145, } \\
\text { Glu166 }\end{array}$ & $\begin{array}{l}\text { Cy3344, His345, Asp368, Arg514, } \\
\text { Tyr515, Arg518 }\end{array}$ \\
\hline $\begin{array}{l}\text { Saikosaponin } \\
\text { A }\end{array}$ & $\begin{array}{l}\text { His41, Glu166, Arg188, } \\
\text { Gln189, Thr190, Gln192 }\end{array}$ & $\begin{array}{l}\text { Ala348, Glu402, Arg514, Tyr515, } \\
\text { Arg518 }\end{array}$ \\
\hline Rutin & $\begin{array}{l}\text { His163, Phe140, Glu166, } \\
\text { Arg188 }\end{array}$ & $\begin{array}{l}\text { Asn149, Arg273, His } 345, \text { Thr445, } \\
\text { His505, Tyr515 }\end{array}$ \\
\hline $\begin{array}{l}\text { Corosolic } \\
\text { acid }\end{array}$ & Gly143, Ser144, Cys145 & Lys363, Thr371 \\
\hline Verbascoside & $\begin{array}{l}\text { Phe140, Gly143, Glu166, } \\
\text { Thr190, Gln192 }\end{array}$ & $\begin{array}{l}\text { Ser128, Glu145, Asn277, Cys344, } \\
\text { His345, Arg518 }\end{array}$ \\
\hline Baicalin & $\begin{array}{l}\text { Thr25, Thr26, Leu141, } \\
\text { Gly143, Ser144, Cys145 }\end{array}$ & $\begin{array}{l}\text { His } 345 \text {, Lys } 363 \text {, Thr371, His } 505 \text {, } \\
\text { Arg5 } 18\end{array}$ \\
\hline Glycyrrhizin & $\begin{array}{l}\text { Phe140, His 163, His164, } \\
\text { Arg188 }\end{array}$ & $\begin{array}{l}\text { Arg273, His } 345, \text { Thr365, Thr371, } \\
\text { Tyr515, Arg518 }\end{array}$ \\
\hline $\begin{array}{l}\text { Mulberroside } \\
\text { A }\end{array}$ & $\begin{array}{l}\text { Thr24, Thr26, Gly143, } \\
\text { Ser144, Cys 145, Gln189 }\end{array}$ & $\begin{array}{l}\text { Asn149, Arg273, Lys363, Asp367, } \\
\text { Asp368, Tyr515, Arg518 }\end{array}$ \\
\hline Cynaroside & Thr24, Thr25, Thr26, Gly143 & Asn149, Pro346, Lys363, Asp368 \\
\hline Bilirubin & $\begin{array}{l}\text { Leu141, Ser144, His } 163 \text {, } \\
\text { Gln189 }\end{array}$ & Thr371, Glu406, Tyr515 \\
\hline
\end{tabular}


Table 5. The structure, natural source and content of active components, and weight ratio of a herb in Chinese patent drugs

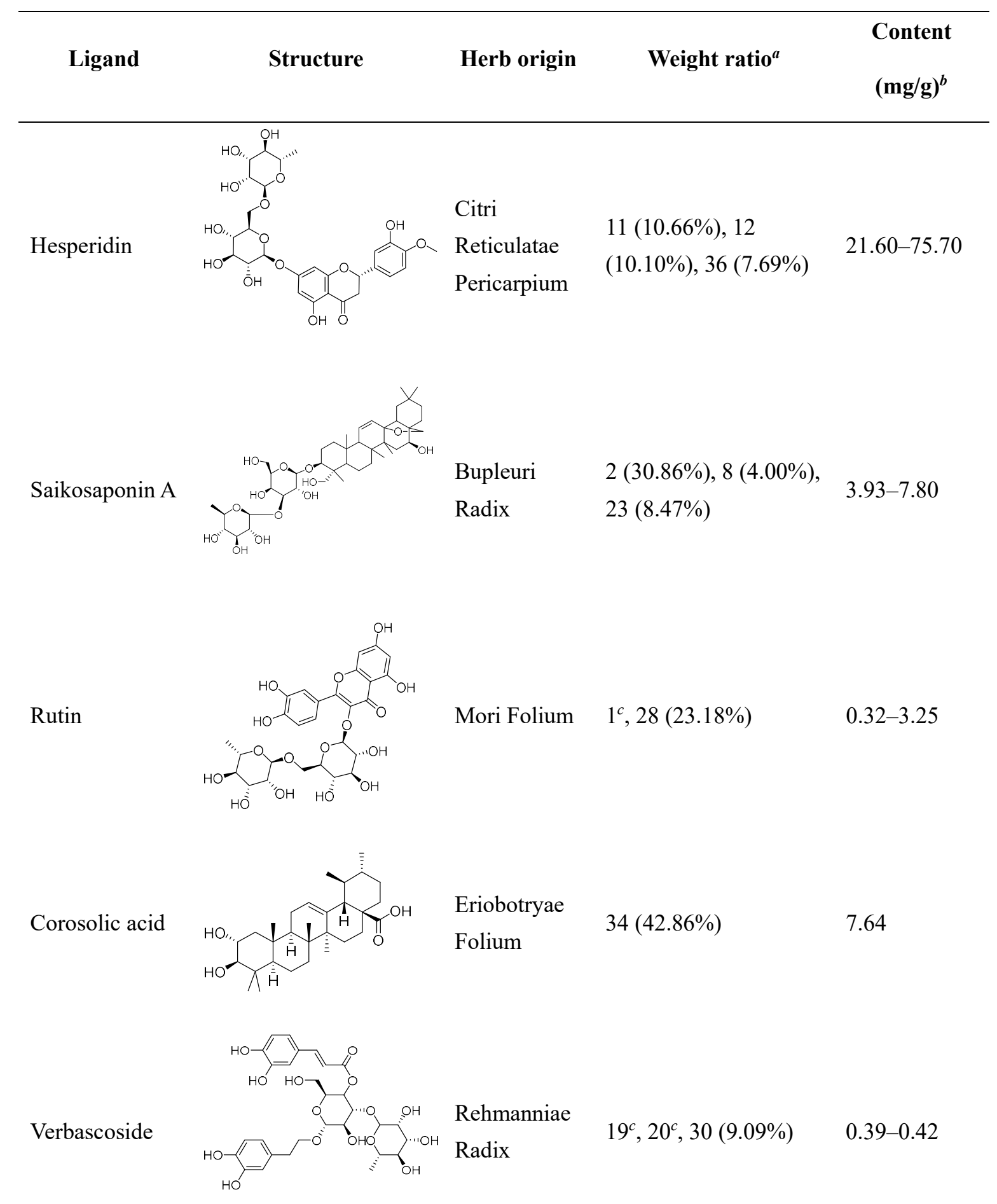


Baicalin<smiles>O=C(O)[C@H]1O[C@@H](Oc2cc3oc(-c4ccccc4)cc(=O)c3c(O)c2O)[C@H](O)[C@@H](O)[C@H]1O</smiles>

Glycyrrhizin

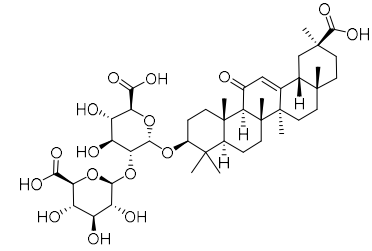

Cynaroside

Bilirubin<smiles></smiles>

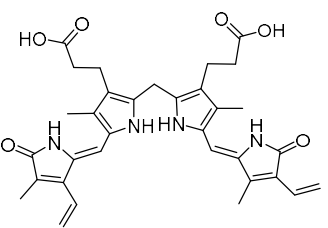

Vincetoxicosid e B

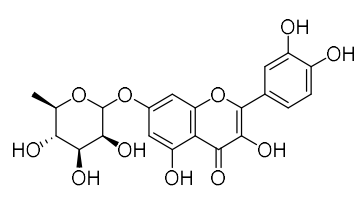

Morusin

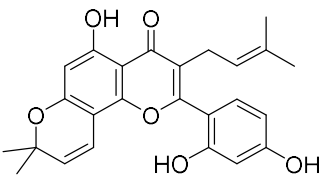

Puerarin

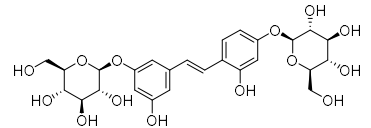

Scutellariae

Radix

Glycyrrhizae

Radix et

Rhizome

Mori

Ramulus

$1^{c}$

4.97-13.14

Lonicerae

Flos

$26(17.01 \%)$

5.1-9.4

Atificial

Cow-bezoar

$38^{c}$

$6.7-9.1$

Cynanchi

Stauntonii

Rhizoma et

$37(5.60 \%)$

No report

Radix

Mori Cortex $37(3.72 \%) \quad 4.40-6.10$

Puerariae $\quad 5^{c}, 8(8.00 \%), 23$

Lobatae

$(8.47 \%), 35(8.57 \%)$,

$11.30-38.93$ radix $\quad 36(11.54 \%)$ 
Orientin

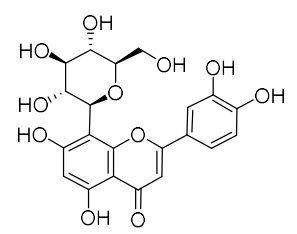

Trollius
Chinensis

$8.56-20.51$

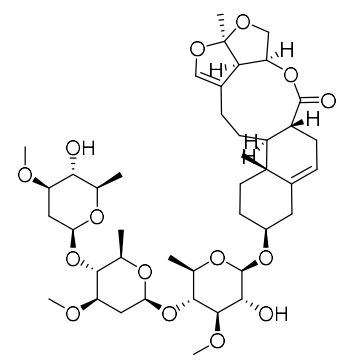

Cynanchi

Atrati Radix $30(9.09 \%)$

$0.04-0.11$ de A

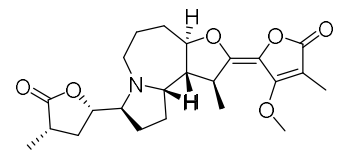

Stemonae

Radix

$37(9.31 \%)$

$2.80-3.80$

Protostemonine<smiles>N#CC(OC1CC(O)C(O)[C@H](O)C1COC1CC(CO)C(O)C(O)C1O)c1ccccc1</smiles>

Armeniacae

$1^{c}, 6^{c}, 13(16.00 \%), 22$

Amygdalin

Semen

(8.82\%), 23 (6.78\%),

Amarum

35 (11.43\%), 36

$36.7-45.8$

(11.54\%)

Ilexgenin A

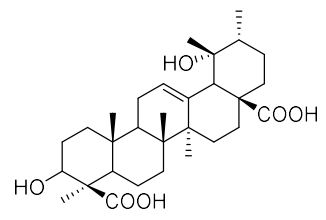

Ilicis

Pubescentis

Radix et

$5^{c}$

4.1-15.6

Caulis

$8(4.00 \%), 9(3.77 \%)$,

Prim- $O$-glucos<smiles>COc1c2oc3cc4c(c(OC)c1c(=O)cc-2o3)O[C@H](CO)[C@@H](C(C)(C)C)O4</smiles>

Saposhnikovi

$22(8.82 \%), 23$

ae Radix

$(8.47 \%), 35$ (5.71\%),

$1.16-9.49$

ylcimifugin

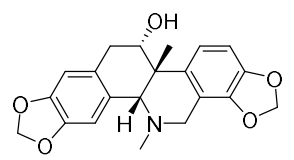

Corydalis

Bungeanae

$23(16.95 \%)$

$1.93-5.80$

Herba

Astragaloside

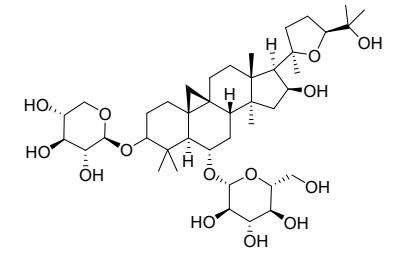

Astragali

Radix

$8(8.00 \%)$

$0.26-2.13$ 
Paeoniae Radix Rubra:

Paeoniflorin

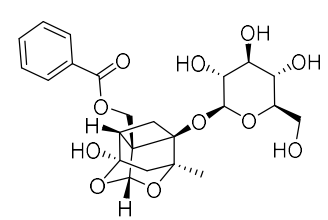

Paeoniae $\quad 8(4.00 \%), 17$

Radix Rubra (42.87\%)

$20.00-25.00$

Paeoniae Paeoniae Radix Alba:

Radix Alba $9(3.77 \%), 22$

(14.70\%)

Polyphyllin I

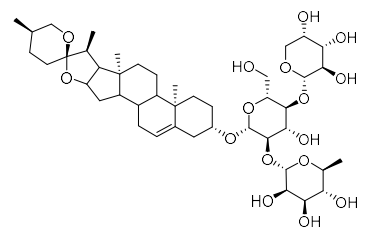

Paridis

Rhizoma

$18^{c}$

$2.87-10.10$

Nodakenin<smiles>C=C1C=Cc2cc3c(cc2O1)OC(C)(OC1OC(CO)[C@H](Cl)C(O)C1O)C3</smiles>

Notopterygii

Rhizoma Et

$5^{c}, 8(6.00 \%), 16^{c}, 35$

Radix

(5.71\%)

$0.50-28.60$

Tectoridin<smiles>COc1cc2c(c(O)c1C)C(C(=O)O)C(c1ccc(O)cc1)CO2</smiles>

Iridis Tectori

Rhizoma

$18^{c}$

$33.27-58.63$

Ursolic acid

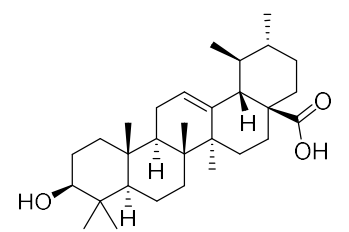

Prunellae

Spica

$28(66.22 \%)$

$2.21-4.15$

Lophatheri

$8(8.00 \%), 25$ (7.14\%),

Herba

26, $33(7.14 \%)$

$0.31-2.29$

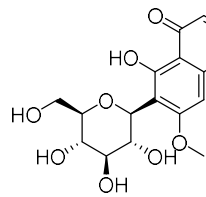<smiles>COc1ccc2cc3c(cc2c1OC)[N+]1=C(CC1)c1cc2c(cc1-2)OCO3</smiles>

Phellodendr

Berberine

i Chinensis $\quad 7^{c}$

17.76-80.32

Cortex

Anemarrhena

e Rhizoma

$19^{c}, 20^{c}$

50.00-92.50

BII

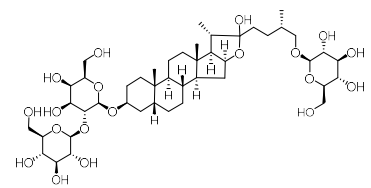


Dryocrassin

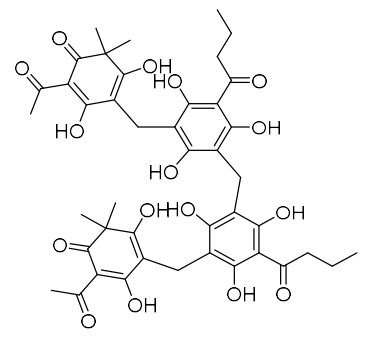

Dryopteridis

Crassirhizom $\quad 6^{c}, 17(14.27 \%) \quad 26.10-61.00$

atis Rhizoma

Columbianadin<smiles>C/C=C(/C)C(=O)OC(C)(C)C1Cc2c(ccc3ccc(=O)oc23)O1</smiles>

Angelicae

Pubescentis

$22(5.88 \%)$

$0.58-7.78$

Radix

Arctiin<smiles>COc1ccc(CC2CCC(=O)C2Cc2ccc(OC3OC(CO)[C@@H](O)C(O)C3O)c(OC)c2)cc1OC</smiles>

Arctii

$1^{c}, 8(8.00 \%), 25$

Fructus

(10.71\%), 26

$47.5-73.3$

(10.21\%), 33 (10.71\%)

Oleanic acid

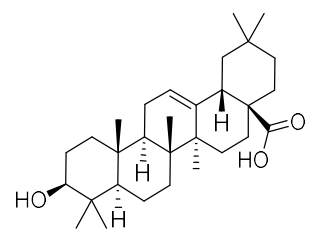

Helictercs

$16^{c}$

$10.18-25.58$

Angustifolia<smiles>O=c1cc(-c2ccc(O)c(O)c2)oc2cc(O)cc(O)c12</smiles>

Lonicerae

Luteolin

Japonicae

$18^{c}$

$2.35-2.45$

Caulis<smiles>O=c1c(O)c(-c2ccc(O)c(O)c2)oc2cc(O)cc(O)c12</smiles>

Desmodium

$16^{c}$

No report

Quercetin

Forsythiaside A

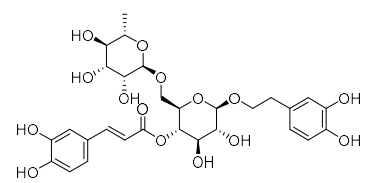

Forsythiae

Fructus

$1^{c}, 6^{c}, 8(6.00 \%), 9$

(3.77\%), 10 (25.00\%),

$19^{c}, 20^{c}, 24(42.86 \%)$,

$25(17.86 \%), 26$

$146.2-172.1$

(17.01\%), 27c, 30

(9.09\%), 33 (17.86\%),

$34^{c}$ 
Radix isatidis

A

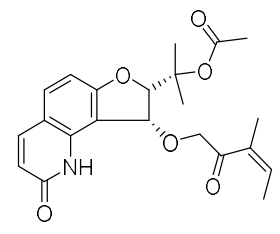

Genistein

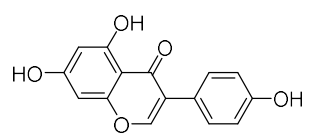

Indirubin

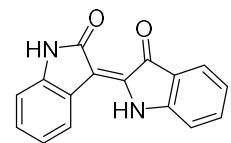

Curcumin<smiles>COc1cc(/C=C/C(=O)CC(=O)/C=C/c2ccc(O)c(OC)c2)ccc1O</smiles>

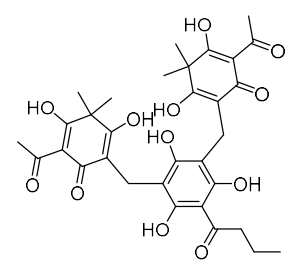

Artemisinin<smiles></smiles>

Emodin<smiles>Cc1cc(O)c2c(c1)C(=O)c1cc(O)cc(O)c1C2=O</smiles>

Cholic acid

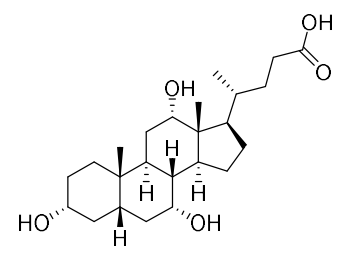

Hyodeoxycholi c acid

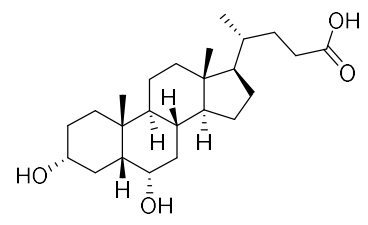

Daidzein
Radix

Isatidis

Radix

Sojae Semen

$25(8.93 \%), 26$

Praeparatum

(8.51\%), 33 (8.93\%)

Isatidis

$8^{c}, 21(60.00 \%), 30$

Folium

(15.15\%)

$0.02-4.15$

Dryopteris

Setosa

$18^{c}$

17.8

Artemisiae

Annuae

$5^{c}$

1.91-5.19

Herba

Rhei Radix

Et Rhizoma

$6^{c}, 9(3.77 \%)$

$0.29-0.66$
$0.84-1.12$

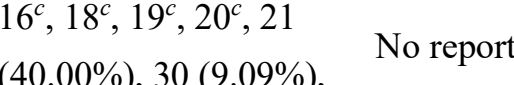

$31(100 \%), 32^{c}$

$19^{c}, 20^{c}$

$0.84-1.12$

Cholic acid $\quad 3^{c}, 32^{c}$

Hyodeoxych $\quad 3^{c}, 32^{c}$
olic acid

Sojae Semen $25(8.93 \%), 26$

Praeparatum $\quad(8.51 \%), 33(8.93 \%)$ 
Xanthiside

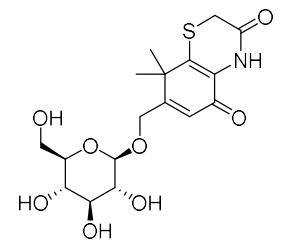

Chlorogenic acid

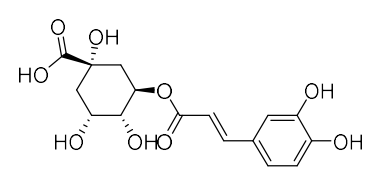

Verbenalin<smiles>COC(=O)C1=COC2C(CC3OC(CO)C(O)C(O)C3O)C3C(=O)CC(C)C3C12</smiles>

Poricoic acid A

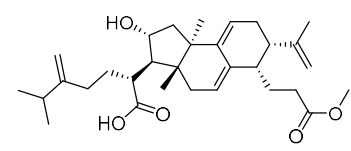<smiles>C=C1CC[C@]2(C(O)O)[C@@H](C)C(=O)[C@H](O)CC[C@@]12C</smiles>

Flos: $3^{c}, 6^{c}, 10$

Lonicerae

(25.00\%), 17

Japonicae

(42.87\%), 24 de

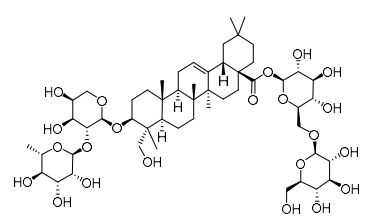

Lonicerae

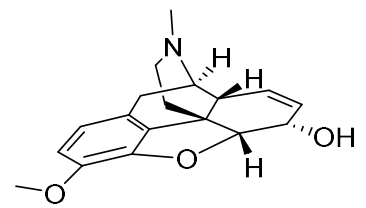

Poria

Andrographi s Herba

$16^{c}$

$11(16.00 \%), 12$ (5.05\%)

Flos

(42.86\%), 25

$(17.86 \%), 27^{c}, 32^{c}, 33$

(17.86\%), $34^{c}$

Chrysanthemi Flos: 28

(10.59\%), 30 (9.09\%)

Verbenae

Herba $5^{c}$

$1.52-3.35$

Flos

Papaveris

Pericarpium

22.30-31.60;

$2.38-7.20$

$0.24-0.40$

$14.9-17.2$

$26(17.01 \%)$

4.30-9.30

$0.23-0.60$

Prunellae

Spica<smiles>CC(CC(Cc1ccc(O)c(O)c1)C(=O)O)C(=O)O</smiles> 
Notopterol

Harpagide

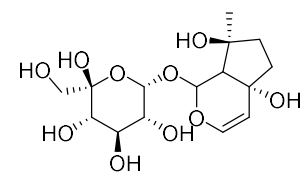

Imperatorin

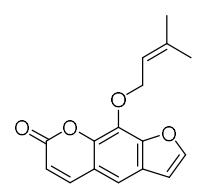

Papaverine

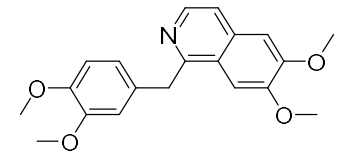

Geniposide<smiles>C=CC(=O)C1=COC(OC2OC(O)C(O)C(O)C2O)C2CC=C(CO)C12</smiles>

Catalpol

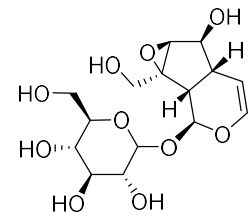

Salidroside<smiles>OCC1OC(OCCc2ccc(O)cc2)C(O)C(O)[C@@H]1O</smiles>

Morphine<smiles>CN1CCC23c4c(ccc(O)c4[C@H](O)C[C@H]12)CCN3C</smiles>

Atractylenolide I

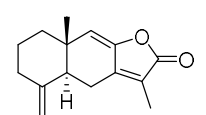

Notopterygii

Rhizoma Et $\quad 5^{c}, 16,35(5.71 \%) \quad 3.50-15.00$

Radix

Scrophularia $8(8.00 \%)$

$3.59-4.86$

Angelicae

$8(4.00 \%), 11$

Dahuricae

(16.00\%), $12(5.05 \%)$,

$18^{c}, 23(5.08 \%), 35$

Radix

$(5.71 \%), 36(7.69 \%)$

Papaveris

Pericarpium

$37(31.05 \%)$

$0.10-0.33$

Gardeniae

Fructus

$3^{c}, 7^{c}, 9(1.89 \%), 32^{c}$

26.25-60.28

Rehmanniae

Radix

$19^{c}, 20^{c} 30(9.09 \%)$

$2.03-11.40$

Rhodiolae

Crenulatae

Radix Et

$6^{c}$

7.83-11.09

Rhizoma

Papaveris

Pericarpium

$37(31.05 \%)$

$0.32-0.93$

Atractylodis

Macrocephal

$9(1.89 \%), 12$

ae Rhizoma
(10.10\%)

1.93-2.54 
Magnolol<smiles>C=CCc1ccc(O)c(-c2cc(CC=C)ccc2O)c1</smiles>

Lobetyolin<smiles>C/C=C/C#CC#CC(O)C(/C=C/CCCO)COC1CC(CO)[C@H](C)C(O)C1O</smiles>

Matrine<smiles>O=C1CCC[C@@H]2C3CCCN4CCCC(CN12)C34</smiles>

Pterodontic acid

Isoevodionol<smiles>COc1cc2c(c(O)c1C(C)=O)C=CC(C)(C)O2</smiles>

Evodia Lepta $16^{c}$

Esculetin<smiles>O=c1ccc2cc(O)c(O)cc2o1</smiles>

Violae Herba

$15^{c}$

$2.02-3.67$

$8(6.00 \%), 9(7.55 \%)$

$12(10.10 \%), 22$

(5.88\%), 23 (5.08\%),

Platycodin D

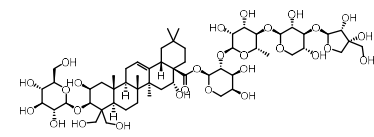

Platycodonis

Radix

25 (10.71\%), 26

(10.21\%), 33

$1.85-4.06$

(10.71\%), 35 (5.71\%),

$36(7.69 \%), 37$

$(3.72 \%)$

Dhelwangin<smiles>Cc1cc(O)c(C(=O)CCC(C)C)c(=O)o1</smiles>

Caffeic acid<smiles>O=C(O)/C=C/c1ccc(O)c(O)c1</smiles>

Lycii Cortex $30(9.09 \%)$<smiles>COc1cc2ccc(=O)oc2cc1O</smiles>

Pogostemoni

s Herba

$6^{c}, 11(5.33 \%), 12$

(9.09\%)

Taraxaci

Herba $15^{c}$

1.84 
Ferulic acid

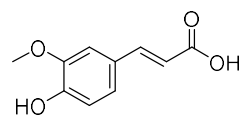

6-Gingerol

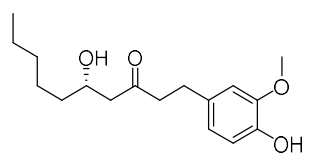

L(+)-Ascorbic acid<smiles>O=C1OC([C@@H](O)CO)C(O)=C1O</smiles>

Atractylodin

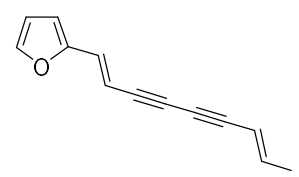

Ephedrine

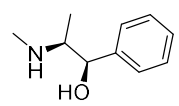

Pulegone

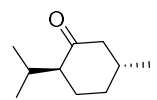

$\alpha$-Asarone<smiles>C/C=C/c1cc(OC)c(OC)cc1OC</smiles>

Coumalic acid<smiles>O=C(O)c1ccc(=O)oc1</smiles>

Citrulline
Phragmitis

Rhizoma

Angelicae

Sinensis

Radix

Zingiberis

Rhizoma

Recens (1.51\%), 22 (5.88\%), $36(7.69 \%)$

Atractylodis Rhizoma

$11(10.66 \%)$

$3.98-10.96$

$13(24.0 \%), 22$

Ephedrae

Herba

(5.88\%), 35 (11.43\%), $36(7.69 \%), 9$ (3.77\%),

$4.80-10.20$ $6^{c}$

$1^{c}, 9(1.89 \%), 23$

Schizonepeta

(16.95\%), 25 (7.14\%)

e Spica

$26(6.80 \%), 27,33$

(7.14\%), 34, 35

$(8.57 \%)$

Acori

Tatarinowii

$19^{c}, 20^{c}, 35(2.86 \%)$

0.84-1.07

Rhizoma

Phragmitis

$1^{c}, 19^{c}, 20^{c}, 23$

Rhizoma

(8.47\%), 26 (10.21\%)

Trichosanthis

Radix
$8(8.00 \%)$

$20.20-60.20$ 
Linolenic acid

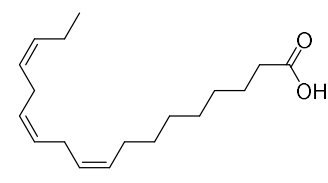

Perillae

Folium

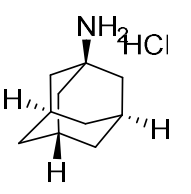

L-Menthol

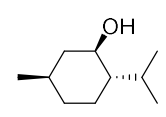

Amantadine

Hydrochloride

$$
\text { H }
$$

Cinnamaldehyd e

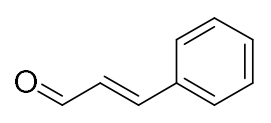

$\beta$-Pinene

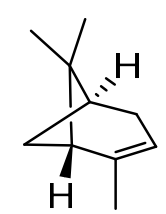

Arecoline<smiles>COC(=O)C1=CCCN(C)C1</smiles>

Glutamic acid<smiles>N[C@@H](CCCC(=O)O)C(=O)O</smiles>

$\alpha-$ Pinene

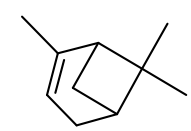

Tetramethyl

pyrazine

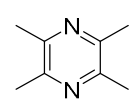

Succinic acid<smiles>O=C(O)CCC(=O)O</smiles>

Decanoy acetaldehyde

Herba

Arecae
$11(2.67 \%), 12$

(5.05\%), $22(5.88 \%), \quad 0.09-0.68$

$23(5.08 \%), 36$

(11.54\%)

$9(3.77 \%), 16^{c}, 23$

Menthae

(5.08\%), 25 (10.71\%),

Haplocalycis

$26(4.73 \%), 27^{c}, 30$

(6.06\%), 33 (10.71\%),

$25.76-226.10$

$34^{c}, 35^{c}, 1^{c}, 37$

$(3.72 \%)$

Cinnamomi $22(8.82 \%), 35$

Ramulus $\quad(8.57 \%), 36(7.69 \%)$

$12.70-16.02$

Forsythiae

$27^{c}, 34^{c}$

$2.40-9.34$

Fructus Oil

$11(16.00 \%), 12$

Pericarpium $\quad(5.05 \%)$

$1.92-3.80$

Bubali Cornu $32^{c}$

Forsythiae

Fructus Oil

$27^{c}, 34^{c}$

$0.75-3.00$

Chuanxiong $35(5.71 \%), 8(4.00 \%)$,

Rhizoma $9(3.77 \%)$

$0.15-0.24$

Pinelliae $\quad 2(11.52 \%), 11$

Rhizoma $\quad(10.66 \%), 12(10.10 \%)$

$3.24-4.43$

Volatile oils

Houttuyniae

$6^{c}, 18^{c}$ 
Taurine<smiles>NCCS(=O)(=O)O</smiles>

Lycii Cortex $30(9.09 \%)$

$3.12-6.20$

Betaine<smiles>C[N+](C)(C)CC(=O)[O-]</smiles>

Lycii Cortex $30(9.09 \%)$ $5.40-10.10$

${ }^{a}$ The number of Chinese patent drugs is same as that in table 1.

${ }^{b}$ Data source: China National Knowledge Infrastructure (CNKI).

${ }^{c}$ Unknown. 